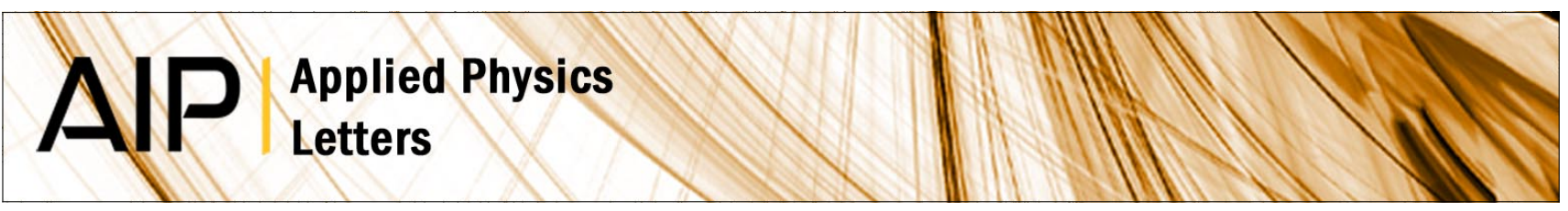

\title{
Decoupling mechanisms and magnetic stability of nanostructured iron chains prepared by sputtering
}

F. Jiménez-Villacorta, E. Céspedes, C. Ocal, and C. Prieto

Citation: Appl. Phys. Lett. 98, 102513 (2011); doi: 10.1063/1.3559918

View online: http://dx.doi.org/10.1063/1.3559918

View Table of Contents: http://apl.aip.org/resource/1/APPLAB/v98/i10

Published by the American Institute of Physics.

\section{Additional information on Appl. Phys. Lett.}

Journal Homepage: http://apl.aip.org/

Journal Information: http://apl.aip.org/about/about_the_journal

Top downloads: http://apl.aip.org/features/most_downloaded

Information for Authors: http://apl.aip.org/authors

\section{ADVERTISEMENT}

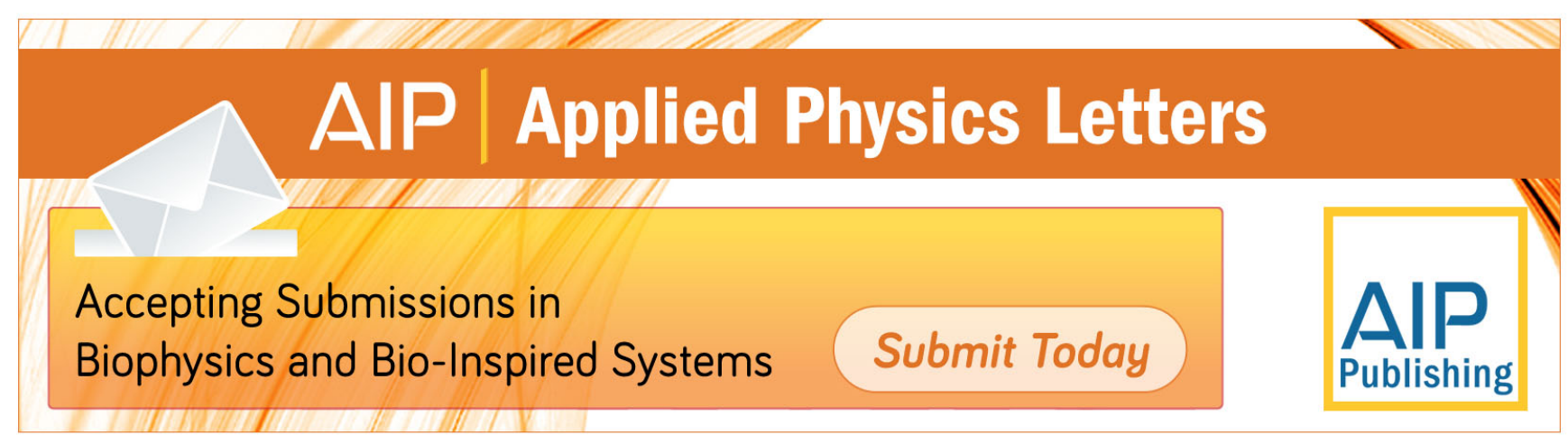




\title{
Decoupling mechanisms and magnetic stability of nanostructured iron chains prepared by sputtering
}

\author{
F. Jiménez-Villacorta, ${ }^{1, a)}$ E. Céspedes, ${ }^{1}$ C. Ocal, ${ }^{2}$ and C. Prieto ${ }^{1, b)}$ \\ ${ }^{1}$ Instituto de Ciencia de Materiales de Madrid, Consejo Superior de Investigaciones Científicas, \\ Cantoblanco, 28049 Madrid, Spain \\ ${ }^{2}$ Instituto de Ciencia de Materiales de Barcelona, Consejo Superior de Investigaciones Científicas, \\ Campus de la UAB, 08193 Bellaterra, Spain
}

(Received 23 December 2010; accepted 2 February 2011; published online 11 March 2011)

\begin{abstract}
Nanostructured $\mathrm{Fe} / \mathrm{Fe}$-oxide systems with a chain morphology formed by interconnected metallic clusters were prepared by sputtering at very low substrate temperatures. The effect of the in situ controlled oxidation at low temperature leads to an effective decoupling of the particles forming the chains, which is attributed to a physical separation of particles through formation of a core-shell metal-oxide structure. In contrast, samples oxidized at room conditions exhibit features of strongly correlated particle systems, in which magnetic decoupling and stabilization can be explained within the framework of the random anisotropy model by considering the effect of the oxide phase. (C) 2011 American Institute of Physics. [doi:10.1063/1.3559918]
\end{abstract}

Recent improvements in the preparation, control, and characterization of nanocomposites, ${ }^{1,2}$ have boosted the interest, for potential applications as magnetic devices, such as sensors, magnetic recording and, in general, magnetic systems with modulated anisotropy. ${ }^{3}$ It is known that properties of magnetic granular arrays ${ }^{4,5}$ and concentrated systems in general are determined not only by the intrinsic particle characteristics, but also by the possible interactions between particle magnetic moments. These interactions may lead to progressive collective behavior, or even clustering, where magnetization reversal and switching of the spins are no longer independent. Such a collective behavior is commonly prevented by covering the particle surfaces with native-oxide shells. ${ }^{6}$

Metal/native-oxide systems obtained by metal deposition and subsequent oxygen exposure $e^{7,8}$ have been proposed as promising materials for their combination of high magnetization and low conductivity, also providing a key response in the enhancement of magnetic stability. Precise manipulation of the particle surface oxidation allows tuning the magnetic and transport properties of core-shell systems through modifications in the spin configuration of metal and oxide phases, ${ }^{9,10}$ with obvious interest for implementation nanoscale magnetoresistive sensors. ${ }^{11}$

In this letter, we report on the magnetic properties of nanostructured Fe systems prepared by sputtering at substrate temperatures far below room temperature. We have found a simple method of preparing magnetic chains with granular morphology by dc-sputtering at temperatures far from the thermodynamical equilibrium. ${ }^{12,13}$ This method is based on previous works in which low temperature deposition of iron thin films induces a magnetic response analog to granular core-shell systems, unlike typical thin film behavior, ${ }^{14}$ providing an interesting approach to the formation and manipulation of the magnetic properties of magnetic nanostructured materials and devices.

\footnotetext{
${ }^{a}$ Present address: Department of Chemical Engineering, Northeastern University, Boston, Massachusetts 02115, USA.

b)Electronic mail: cprieto@icmm.csic.es.
}

In that work, we focus our attention in samples prepared at $\mathrm{T}_{\mathrm{S}}=200 \mathrm{~K}$. To observe the effects of the natural oxidation in the magnetic properties of this type of nanostructured systems, some samples (A-type) were taken from the growth chamber and passivated on air at room temperature. Other samples (B-type) were postgrowth oxidized in situ at the deposition temperature by a controlled process consisting in flowing $\mathrm{O}_{2}$ at a pressure of $1 \times 10^{-1}$ mbar. Nanostructural and compositional characterizations have been carried out for A-type samples; it should be comment that no relevant differences in those aspects are expected between both preparations.

The sample morphology and the conducting response were investigated by a scanning probe microscopy strategy. ${ }^{13}$ Topography and conductive-scanning force microscopy (CSFM) corresponding to an A-type sample are shown in Fig. 1. Samples prepared at such low temperatures present the morphology of interconnected Fe grains, in contrast with the film microstructure of analog samples deposited at room temperature. Most of the grains have an average size of around $12 \mathrm{~nm}$. However, some of the grains have a larger size of about $22 \mathrm{~nm}$. A similar bimodal grain size distribution has been recently observed in nanostructured gold films $(\sim 10 \mathrm{~nm})$ on $\mathrm{SiO}_{2}$ and explained in terms of atomic diffusion on metallic grain boundaries. ${ }^{15}$ Moreover, C-SFM proves that larger grains present metallic character, whereas they form chains connected by grains having larger resistance [Fig. 1(c)] and presenting semiconductor behavior. ${ }^{13}$

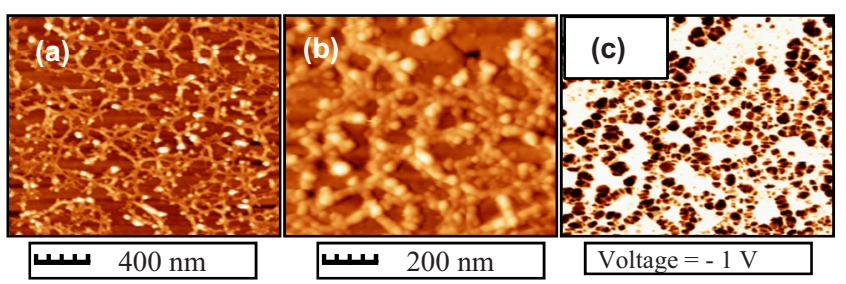

FIG. 1. (Color online) (a) Scanning probe micrograph of a sample prepared at $\mathrm{T}_{\mathrm{S}}=200 \mathrm{~K}$. [(b) and (c)] Topography and conductive micrograph obtained under an applied voltage of $-1 \mathrm{~V}$ between the tip and the sample, black and white colors stand for high and low intensity values, respectively. 

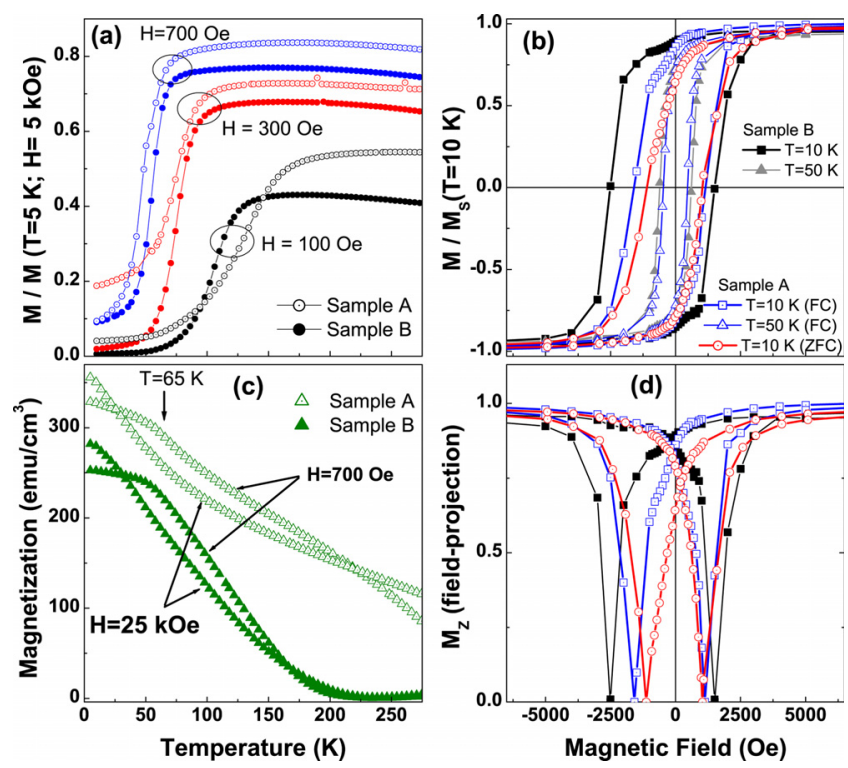

FIG. 2. (Color online) (a) ZFC at intermediate fields of granular Fe chains prepared at $\mathrm{T}_{\mathrm{S}}=200 \mathrm{~K}$; (b) magnetization loops at low temperatures; (c) TRM curves at $\mathrm{H}=700 \mathrm{Oe}$ and $\mathrm{H}=25 \mathrm{kOe}$; and (d) magnetization projection along the applied field in loops at $\mathrm{T}=10 \mathrm{~K}$.

A previous combined analysis by $\mathrm{x}$-ray absorption spectroscopy and magnetic circular dichroism measured at the $\mathrm{FeL}_{2,3}$ edges has revealed that these samples are formed by metallic iron and iron oxides including ferrimagnetic $\mathrm{Fe}_{3} \mathrm{O}_{4}$ and $\gamma-\mathrm{Fe}_{2} \mathrm{O}_{3}$, as well as the antiferromagnetic $\mathrm{FeO}$ phase. ${ }^{16}$

Magnetic measurements were achieved in a superconducting Quantum interference device magnetometer (MPMS-5T from Quantum Design). Zero-field-cooling curves at several intermediate fields for A and B samples are shown in Fig. 2(a). Both samples exhibit granular behavior with strong temperature dependence of the zero-field-cooled (ZFC) curve, which is associated to superspin reorientation. However, for the A-sample the blocking temperature distribution is broader than for the B-sample. This is markedly visible at low fields $(\mathrm{H}=100 \mathrm{Oe})$, where apart from the narrower energy barrier distribution of B sample, an almost constant value of the magnetization below the blocking temperature is seen. Such flattened ZFC curves in the in situ oxidized sample at low temperatures and low fields can be interpreted as a signature of superspin frustration resulting from the effective pinning exerted by the glassy oxide shell at low probing fields and in the low temperature regime. ${ }^{17}$

Magnetization loops at 10 and $50 \mathrm{~K}$ are shown in Fig. 2(b). By comparing ZFC and field-cooled loops (applied cooling field of $20 \mathrm{kOe}$ ) at $\mathrm{T}=10 \mathrm{~K}$ for A-sample, an increasing asymmetry can be observed. Enhanced values of remanence and coercivity in the descending branch are a signature of exchange anisotropy, ${ }^{6}$ due to the pinning effect of the surface spins on the metallic grains in the field-cooled loop. In addition, B-sample exhibits higher values of coercivity and remanence with a noticeable exchange bias $\left(\mathrm{H}_{\mathrm{E}}\right.$ $\approx 500 \mathrm{Oe}$ at $\mathrm{T}=10 \mathrm{~K})$ compared with A-sample $\left(\mathrm{H}_{\mathrm{E}}\right.$ $\approx 200 \mathrm{Oe}$ ). At $\mathrm{T}=50 \mathrm{~K}$, the exchange bias disappears, but the effects of the exchange interaction provided by the oxide phase are still present in terms of an enhanced coercivity.

A further insight in the magnetization reversal mechanisms of the two samples is provided by evaluating the magnetization projection along the field axis, $M_{Z}=|M \cos \theta|$, where $\theta$ is the angle between the virtual total reversal magnetization and the probing field. ${ }^{18,19}$ Competition between magnetic anisotropies determines the asymmetric behavior of the magnetization reversal. Assuming that the main contribution to magnetization signal comes from the metallic cores, remarkable differences are observed in the reversal process for each type of the samples. To have an asses of $M_{Z}$, Fig. 2(d) shows the absolute value of some magnetization loops depicted in Fig. 2(b). Clearly, it can be observed that the decreasing branch peaks of A-sample magnetization loops are broader than the increasing ones, in agreement with strongly correlated superspin systems. Conversely, B-sample presents narrower and more symmetric peaks for both branches, suggesting a one-step reversal process of independent magnetic particles. Moreover, this larger loop shearing at the descending branch of B-sample respect to A-one suggests a weakening of intergranular magnetic coupling, ${ }^{20}$ fostering a uniform rotation in the reversal process of such particles.

Thermoremanence magnetization (TRM) curves at intermediate $(\mathrm{H}=700 \mathrm{Oe})$ and saturating $(\mathrm{H}=25 \mathrm{kOe})$ fields are shown in Fig. 2(c). At intermediate fields, magnetic moments of the particles are not fully oriented. At low temperatures, both samples present a flattened shape, likely related to the pinning effect exerted by the oxide coverage spins. In fact, it should be noted that this effect is stronger in B-sample, in agreement with the larger exchange bias obtained in the low temperature magnetization loops. At $\mathrm{T}>50 \mathrm{~K}$, when the effect of the oxide phase vanishes, the magnetization decay (which is typical from magnetic particle systems) starts for both samples. Differences in the TRM behavior for each type of samples may be summarized in a smoother decrease with increasing temperature for A-sample.

At high saturating fields, the superspin moments are practically aligned to the field at low temperature, even in the remanence state $\left(\approx 0.9 \mathrm{M}_{\mathrm{S}}\right.$, as obtained from the magnetization loops). As in the case of intermediate fields, a similar behavior at low temperatures is observed in both samples. As temperature increases, B-sample presents a continuous decay of remanent magnetization, falling to zero at around $220 \mathrm{~K}$. That decay is typical from noninteracting or very weakly interacting particle systems. This fact suggests that samples oxidized at low temperatures are formed by separated magnetic Fe grains that are surrounded by an oxide phase, like a core-shell structure. Therefore, the oxide phase was formed as well in the interparticle spaces.

TRM curves of A-sample show a different behavior of the superspin thermalization. At $\mathrm{T}>65 \mathrm{~K}$, the decrease in the magnetization when increasing temperature is much smoother than the observed for B-sample in both fields TRM curves. This behavior suggests that strong correlation between particles affects the remanence decay ${ }^{21,22}$ in such a way that magnetization is maintained throughout a wide range of temperatures. In this case, magnetic interparticle interactions indicate that partial oxidation at room conditions does not provide a physical decoupling of the magnetic grains.

Figure 3 schematically illustrates a representative picture of the different physical decoupling of iron particles in Aand B-samples. A qualitative explanation could be found in the large difference of thermal expansion coefficients of iron $\left(\sim 1.3 \times 10^{-5} \mathrm{~K}^{-1}\right)$ and silicon $\left(\sim 3 \times 10^{-6} \mathrm{~K}^{-1}\right)$. Due to 


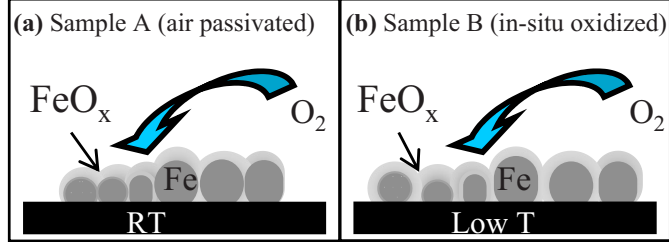

FIG. 3. (Color online) Schematic representation of the oxidation processes of both types of samples. (a) Samples passivated at room conditions and (b) samples oxidized at low temperature.

those different coefficients, during sample heating the resultant compressive strain tends to decrease distances between the iron grains along in-plane directions preventing an easy oxide growth between them at room conditions in comparison with an isotropic oxidation at the low preparation temperature.

However, it is worth to further comment on the similar TRM curves obtained for both types of samples at $\mathrm{T}$ $<65 \mathrm{~K}$, because decoupling of the particles seems to be effective at such temperature range. This observation can be explained within the formalism of the random anisotropy model ${ }^{23}$ accounting for the interaction effects between the metallic clusters and the oxide phase, in terms of increasing anisotropy. In the framework of this model, two competing terms are defined: the anisotropy field and the exchange field. Increasing anisotropy field of the particles may bear a resemblance of separate, though still interacting, particles. ${ }^{24}$ For that reason in A-sample, the pinning of the covering oxide phase spins provides exchange anisotropy added to the uniaxial anisotropy, which yields a behavior analog to that observed for decoupled particles at low temperatures. When increasing temperature, this added anisotropy is no longer effective and the superspins are strongly correlated with their neighboring particles.

In conclusion, we have prepared granular magnetic chains formed by interconnected iron particles harnessing the peculiar features of dc-sputtering deposition at low temperatures. Oxidation processes at low temperatures critically determine the magnetic behavior of the granular chains. Magnetization loops show the differences between samples naturally passivated at room conditions and samples oxidized by flowing $\mathrm{O}_{2}$ in the preparation chamber at the deposition temperature. An enhanced stability of the magnetization is observed for low temperature oxidized samples, which is explained in terms of the stronger driven pinning force exerted by the oxide phase spins. Magnetothermal curves reveal the granular magnetic behavior of such low $\mathrm{T}_{\mathrm{S}}$ samples, suggesting that in situ oxidation at the low deposition temperature provides an effective decoupling of the particle moments. Such effective decoupling and magnetic stability can be explained by the formation of a nonferromagnetic oxide phase between the particles, creating a "physical" separation or isolation of the iron nanoparticles. Nevertheless, the covering oxide phase adds an extra anisotropy at low temperature in both types of samples, yielding a "magnetic" separation between the particle superspins. As a final remark, it should be noted that this simple preparation process allows the tuning of the magnetic features of these nanoparticle, which enable these systems for future applications in the fabrication of magnetically enhanced granular systems for advanced magnetic devices.

This work has been supported by Spanish MICINN under Contract Nos. MAT2009-08786 and CSD2009-00013 as well as by the Madrid Regional Government through Contract No. S2009/MAT-1756. We, specially, acknowledge C. Munuera for AFM experiments.

${ }^{1}$ J. R. Morales, S. Tanju, W. P. Beyermann, and J. E. Garay, Appl. Phys. Lett. 96, 013102 (2010).

${ }^{2}$ P. Gorria, M. P. Fernández-García, M. Sevilla, J. A. Blanco, and A. B. Fuertes, Phys. Status Solidi (RRL) 3, 4 (2009).

${ }^{3}$ A. Breitling, T. Bublat, and D. Goll, Phys. Status Solidi (RRL) 3, 130 (2009).

${ }^{4}$ K. Nakata, Y. Hu, O. Uzun, O. Bakr, and F. Stellacci, Adv. Mater. (Weinheim, Ger.) 20, 4294 (2008).

${ }^{5}$ B. Y. Geng, J. Z. Ma, X. W. Liu, Q. B. Du, M. G. Kong, and L. D. Zhang, Appl. Phys. Lett. 90, 043120 (2007).

${ }^{6}$ V. Skumryev, S. Stoyanov, Y. Zhang, G. Hadjipanayis, D. Givord, and J. Nogués, Nature (London) 423, 850 (2003).

${ }^{7}$ S. Couet, K. Schlage, K. Saksl, and R. Röhlsberger, Phys. Rev. Lett. 101, 056101 (2008).

${ }^{8}$ G. S. D. Beach, F. T. Parker, D. J. Smith, P. A. Crozier, and A. E. Berkowitz, Phys. Rev. Lett. 91, 267201 (2003).

${ }^{9}$ S. E. Inderhees, J. A. Borchers, K. S. Green, M. S. Kim, K. Sun, G. L. Strycker, and M. C. Aronson, Phys. Rev. Lett. 101, 117202 (2008).

${ }^{10}$ J. S. Hwang, L. W. Chen, T. C. Chen, C. W. Kuo, Z. S. Hu, T. R. Tsai, Y. J. Wu, T. Y. Lin, Y. Y. Jhuo, C. Y. Cheng, C. M. Lin, and Y. H. Liu, Nanotechnology 20, 055302 (2009).

${ }^{11}$ C. Martínez-Boubeta, L. Balcells, C. Monty, P. Ordejón, and B. Martínez, Appl. Phys. Lett. 94, 062507 (2009).

${ }^{12}$ L. Gránásy, T. Pusztai, T. Börzsöni, J. A. Warren, and J. F. Douglas, Nature Mater. 3, 645 (2004).

${ }^{13}$ F. Jiménez-Villacorta, C. Munuera, C. Ocal, and C. Prieto, J. Nanopart. Res. 12, 1117 (2010).

${ }^{14}$ F. Jiménez-Villacorta, Y. Huttel, A. Muñoz-Martín, C. Ballesteros, E. Román, and C. Prieto, J. Appl. Phys. 101, 113914 (2007).

${ }^{15}$ F. Ruffino, M. G. Grimaldi, C. Bongiorno, F. Giannazzo, F. Roccaforte, V. Raineri, and C. Spinella, J. Appl. Phys. 105, 054311 (2009).

${ }^{16} \mathrm{~F}$. Jimenez-Villacorta (unpublished).

${ }^{17}$ D. L. Peng, K. Sumiyama, T. Hihara, S. Yamamuro, and T. J. Konno, Phys. Rev. B 61, 3103 (2000).

${ }^{18}$ O. Iglesias, A. Labarta, and X. Batlle, J. Nanosci. Nanotechnol. 8, 2761 (2008).

${ }^{19}$ F. Jiménez-Villacorta and C. Prieto, J. Phys.: Condens. Matter 20, 085216 (2008).

${ }^{20}$ B. M. Lairson, W. Liu, A. P. Payne, C. Baldwin, and H. Hamilton, J. Appl. Phys. 77, 6675 (1995).

${ }^{21}$ R. W. Chantrell, N. Walmsley, J. Gore, and M. Maylin, Phys. Rev. B 63, 024410 (2000).

${ }^{22}$ A. Slawska-Waniewska, A. Roig, M. Gich, L. Casas, K. Raka, N Nedelko, and E. Molins, Phys. Rev. B 70, 054412 (2004).

${ }^{23}$ E. Chudnovsky, W. M. Saslow, and R. A. Serota, Phys. Rev. B 33, 251 (1986).

${ }^{24}$ C. Binns and M. J. Maher, New J. Phys. 4, 85 (2002). 